\title{
Inner perturbations in noncommutative geometry
}

\author{
Walter van Suijlekom* \\ Radboud University Nijmegen - IMAPP (Mathematics) \\ E-mail: waltervs@math.ru.nl
}

\begin{abstract}
Starting with an algebra, we define a semigroup which extends the group of invertible elements in that algebra. As we will explain, this semigroup describes inner perturbations of noncommutative manifolds, and has applications to gauge theories in physics. We will present some elementary examples of the semigroup associated to matrix algebras, and to (smooth) functions on a manifold. Joint work with Ali Chamseddine and Alain Connes.

References
\end{abstract}

[1] A.H. Chamseddine, A. Connes, and W. D. Van Suijlekom. "Inner fluctuations in noncommutative geometry without the first order condition", J. Geom. Phys. 73 (2013) 222-234.

[2] A.H. Chamseddine, A. Connes, and W. D. van Suijlekom. "Beyond the spectral Standard Model: Emergence of Pati-Salam unification”, JHEP 1311 (2013) 132.

Frontiers of Fundamental Physics 14 - FFP14,

15-18 July 2014

Aix Marseille University (AMU) Saint-Charles Campus, Marseille

\footnotetext{
*Speaker.
} 